\title{
The Effect of Fixing Method on the Result of Ultrasonic Infrared Thermography Testing
}

\author{
Gan Tian ${ }^{1}$, Fazhong $\mathrm{Li}^{2}$, Shuaixing Feng ${ }^{3}$, Haohao Wan², Guangjie $\mathrm{Kou}^{2}{ }^{2}{ }^{*}$ \\ ${ }^{1}$ Rocket Force University of Engineering, $710025 \mathrm{Xi}$ 'an Shaanxi, China \\ ${ }^{2}$ Project Management Center of PLA Rocket Force Equipment Department, 100085 Beijing, China \\ ${ }^{3}$ The $41^{\text {st }}$ Institute of The Sixth Academy of China Aerospace Science \& Industry Corporation, 010010 Huhehaote Inner Mongolia \\ Autonomous Region, China
}

\begin{abstract}
Considering the influence of the fixing method of the component in the system on its results in ultrasonic infrared thermography testing, the detection experiments of the cracked plat structure with fixing at one end and fixing at two ends are carried out through different fixing methods of the equivalent structure. The results show that for the same structure to be detected, the more the bounds are fixed, the more the degrees of freedom are restricted, the less ultrasonic energy is dissipated during the inspection process, and the better the inspection effect. The work has positive significance for the selection of detection parameters in ultrasonic infrared thermography testing, helpful to provide some references for it.
\end{abstract}

\section{Introduction}

In recent years, safety accidents caused by micro-cracks, fatigue and fracture of key structures are not uncommon. For example, the windshield burst of Sichuan Airlines Flight 8633 caused a moment of fright [1], the fatigue crack of the US F15C fighter jet's nose caused air disintegration [2], and the US $\mathrm{KC}$ the propeller of the $130 \mathrm{~T}$ transport/tanker broke in the air, causing the plane to crash [3]. During the operation of equipment, some key structures need to withstand external loads such as pressure, temperature, corrosion, or alternating stress during service, which are prone to cracks, fatigue and other damages, which can lead to structural fractures in severe cases, bringing serious hidden dangers to equipment safety. Therefore, it is very important to conduct non-destructive testing of its key structures during the service process of the equipment [4-7]. Ultrasonic infrared thermography testing technology is an emerging non-destructive testing technology in recent years [8]. It has the characteristic of selective heating of defects, especially suitable for damage detection of complex structures and micro-cracks [9][10]. The detection process of ultrasonic infrared thermography testing is mainly composed of four parts: heat generation in the defect area under power ultrasonic excitation, heat flow conduction, surface temperature field acquisition, and heat map recognition and processing [11][12].

In actual detection, the inspection results of ultrasonic infrared thermography testing are easily affected by the fixing method of the inspected structure. Therefore, to realize the real-time inspection of key structures, it is necessary to analyse the influence of the component fixing method on the crack heat generation.
This is helpful to select the detection parameters under the external conditions to achieve good detection results.

\section{Fundamental theory}

In the process of ultrasonic infrared thermal imaging detection, under the action of power ultrasonic waves, the test sample is forced to vibrate, causing frictioncollision of the defect interface and generating heat. The specimen can be regarded as a multi-degree-of-freedom dynamic system during the inspection process, and the transient dynamic equation of its forced vibration is:

$$
\boldsymbol{M} \frac{d^{2} \boldsymbol{U}}{d t^{2}}+\boldsymbol{C} \frac{d \boldsymbol{U}}{d t}+\boldsymbol{K} \boldsymbol{U}=\boldsymbol{F}+\boldsymbol{R}
$$

Where $\boldsymbol{M}$ is the mass matrix, $\boldsymbol{C}$ is the damping matrix, $\boldsymbol{K}$ is the stiffness matrix, $\boldsymbol{U}$ is the nodal displacement matrix, $\boldsymbol{F}$ is the external load matrix, $\boldsymbol{R}$ is the crack interface contact force matrix, and $t$ is the excitation time.

In this process, due to the small proportions of plastic deformation heat generation and damping heat generation, only considering friction heat generation, the heat flow $\mathrm{q}(\mathrm{t})$ generated by the defect interface frictioncollision is [13]:

$$
q(t)=\gamma \cdot\left[\mu_{\mathrm{d}}+\left(\mu_{\mathrm{s}}-\mu_{\mathrm{d}}\right) e^{-\beta|v|}\right] R_{N}(t) \cdot v_{T}(t)
$$

Where, $\gamma$ is the energy conversion efficiency, $R_{N}(t)$ is the element contact force, $v_{T}(t)$ is the node velocity in the defect area, $\mu_{\mathrm{d}}$ is the dynamic friction coefficient, $\mu_{\mathrm{s}}$ is the static friction coefficient, $\beta$ is the static friction converted to the dynamic friction coefficient, and $v$ is the slip rate. When the excitation time lasts for $\mathrm{ts}$, the heat generated by the defect area $W(\tau)$ can be expressed as:

\footnotetext{
*Corresponding author: 18119342611@163.com
} 


$$
W(\tau)=\int_{0}^{\tau} q(t) d t
$$

Equation (1)-(3) show the basic process of heat generation in the defect area under ultrasonic excitation, and shows the feasibility of ultrasonic infrared thermal imaging detection.

\section{Experiment method}

\subsection{Principle}

However, theoretical analysis also has some limitations. In the actual testing process, there are many ways to install components and systems.

Fig. 1 shows four different types of parts on the aircraft. The red circle or ellipse marks the position of the structure in the system. It can be seen that there is only one end connection between the vertical tail and the fuselage in Fig. 1(a), so the connection method can be simplified as a cantilever beam, and the simulation is clamped by one end in Fig. 2(a). The two ends of the pipe in Fig. 1(b) connected to the engine, the connection method can be simplified as fixing at two ends, using Fig. 2(b) to simulate the two ends are clamped. In Fig. 1(c), when installing the engine blade, all three ends of the blade root should be fixed to the blisk, and the simulation is clamped by three ends in Fig. 2(c). When analysing a small part of the area on the shell in Fig. 1(d), it can be found that the connection between this area and the system is all fixed, that is, the four ends are fixed, using Fig. 2. (d) to simulate.

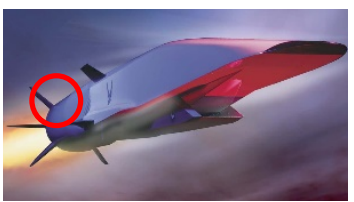

(a)

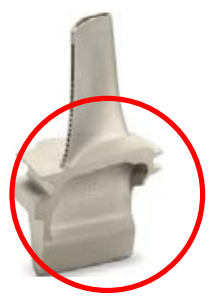

(c)

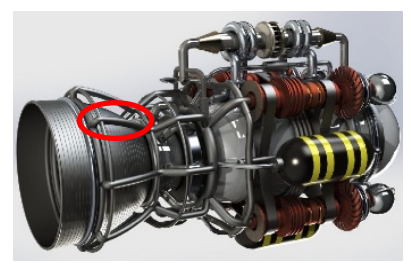

(b)

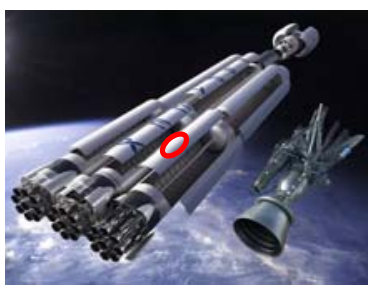

(d)
Fig. 1. Different fixing methods of components: (a) Cantilever beam, (b) Fixed at two ends, (c) Three-terminal fixed and (d) All fixed (a)

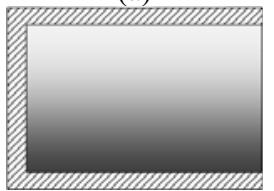

(c)

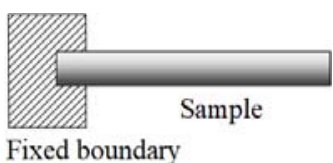

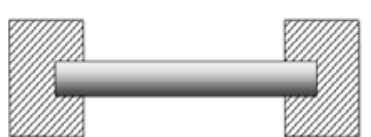

(b)

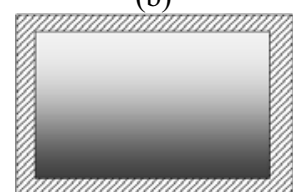

(d)
Fig. 2. Diagram of different fixing methods: (a) Fixed at one end, (b) Fixed at two ends, (c) Fixed at three ends and (d) All fixed

Therefore, one end is clamped to simulate the cantilever beam component, the two ends is clamped to simulate the two-end fixed component, the three ends is clamped to simulate the three-terminal fixed component, and the four ends is clamped to simulate all fixed (complete installation) components.

\subsection{Device and sample}

Figure 3 shows an ultrasonic infrared thermography testing platform with adjustable specimen fixing methods. The platform is composed of an infrared camera, an ultrasonic horn, an ultrasonic controller, and a computer system. The excitation frequency is not adjustable, and the maximum excitation amplitude is $70 \mu \mathrm{m}$. The excitation amplitude percentage and excitation time can be adjusted by setting the parameters of the ultrasonic controller.

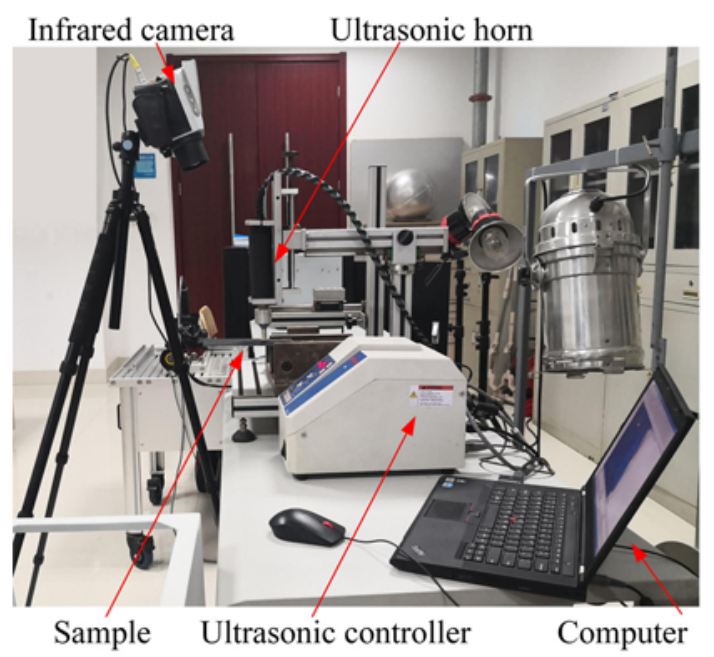

Fig. 3. Ultrasonic infrared thermography testing platform

Fig. 4 shows the geometric characteristics of the $1 \#$ and $2 \#$ samples respectively. The geometric dimensions are $150 \mathrm{~mm} \times 150 \mathrm{~mm} \times 5 \mathrm{~mm}$. The crack length of the $1 \#$ sample is about $40 \mathrm{~mm}$, which is relatively tortuous. Due to the large gap in the crack interface, the crack is easy for visual inspection. However, the crack of $2 \#$ sample is only $30 \mathrm{~mm}$, the visual inspection is very difficult because the gap in crack interface is very close. 


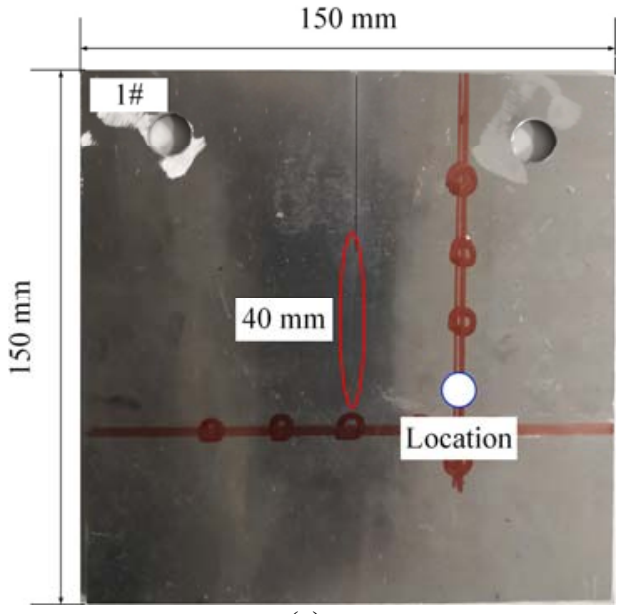

(a)

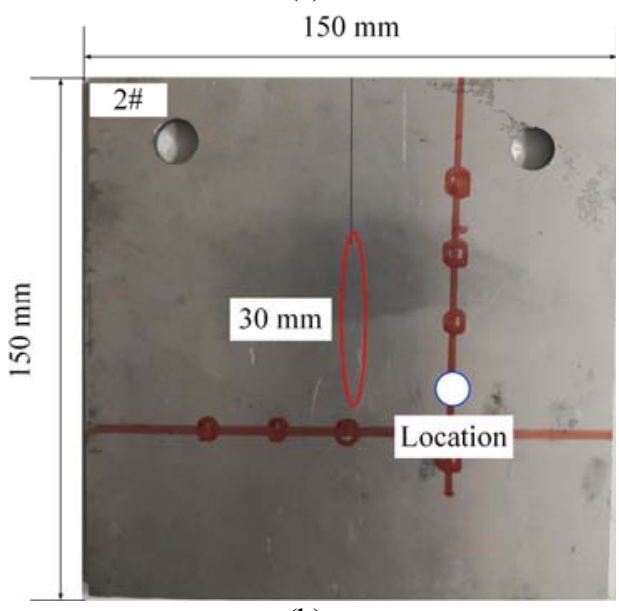

(b)

Fig. 4. Geometric characteristics of the tested samples: (a) 1\# and (b) $2 \#$

The physical diagram of fixing at one end and fixing at two ends are shown in Fig. 5. Firstly, 1\# sample is adjusted to one end mode, the excitation amplitude is set to $100 \%$, the excitation time is set to $0.3 \mathrm{~s}$, and the excitation position is as the corresponding position on the back of the mark. After the testing is completed,

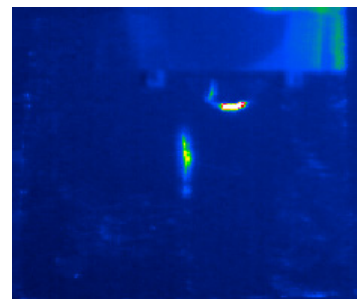

(a)

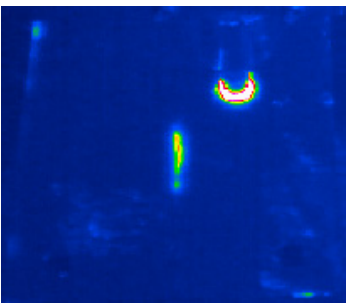

(b)
Fig. 6. Physical map of different fixing methods: (a) Fixed at one end and (b) Fixed at two ends

In order to analyse the above results more specifically, the temperature change at the maximum point of the crack area temperature rise is extracted, and the crack area temperature rise curves of the two samples in different fixing modes are drawn, as shown in Fig. 8.

It can be seen that the maximum temperature rise of 1 \# sample is about $6.2^{\circ} \mathrm{C}$ when fixed at two ends, and the maximum temperature rise is about $5.7^{\circ} \mathrm{C}$ when one end testing the $2 \#$ sample, the fixing methods of $1 \#$ and the $2 \#$ are changed as fixing at two ends, and other parameters remain unchanged to carry out the testing.

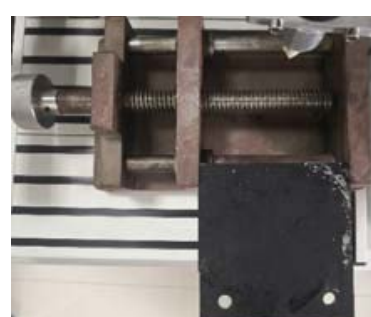

(a)

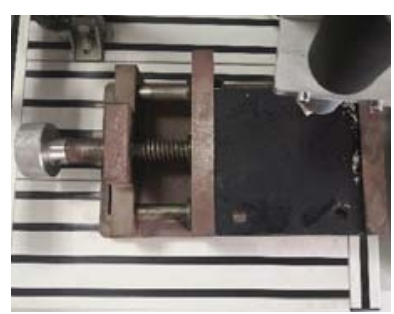

(b)
Fig. 5. Physical map of different fixing methods: (a) Fixed at one end and (b) Fixed at two ends

\section{Results and discussions}

After the experiment, the surface temperature distributions of the samples are depicted in Fig. 6 and Fig. 7. It can be found that, for the $1 \#$ and $2 \#$ samples, under the condition of fixing at two ends, the hot spots in the crack area are obviously brighter than the case where one end is fixed. It is easier to detect defects in the structure when the two ends are fixed. The results show that the more complete the component is installed and fixed in the system, the easier it is to detect defects. This is because the more the boundary of the component is fixed in the system, the smaller the vibration energy loss during ultrasonic excitation, so that more ultrasonic energy is converted into heat energy at the defect, and the detection effect is better, and the defect hot spot more obvious. On the other hand, because the crack of $1 \#$ sample is $10 \mathrm{~mm}$ longer than $2 \#$ sample, under ultrasonic excitation, the frictional collision energy dissipation of the crack contact interface is more than that of $2 \#$ sample, and the hot spot is brighter than $2 \#$ sample.

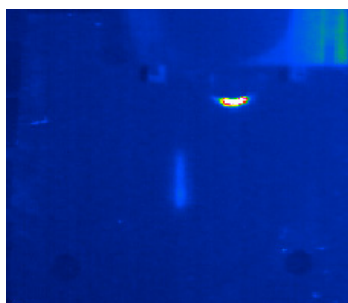

(a)

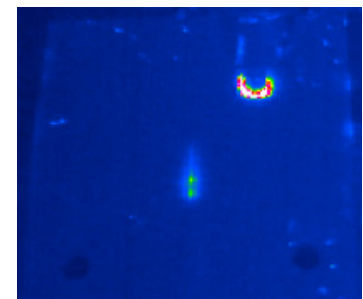

(b)
Fig. 7. Physical map of different fixing methods: (a) Fixed at one end and (b) Fixed at two ends

is fixed; the maximum temperature rise of $2 \#$ sample is about $4.2^{\circ} \mathrm{C}$ when fixed at two ends, when one end is fixed, it is about $1.5^{\circ} \mathrm{C}$. Although the maximum temperature rise difference between fixed at one end and fixed at two ends of the $1 \#$ sample is small, it can be seen that the difference in hot spot area and brightness under the two fixing methods is relatively large. It 
indicates that the ability of cracks in ultrasonic infrared thermography testing is better than fixing at one end.

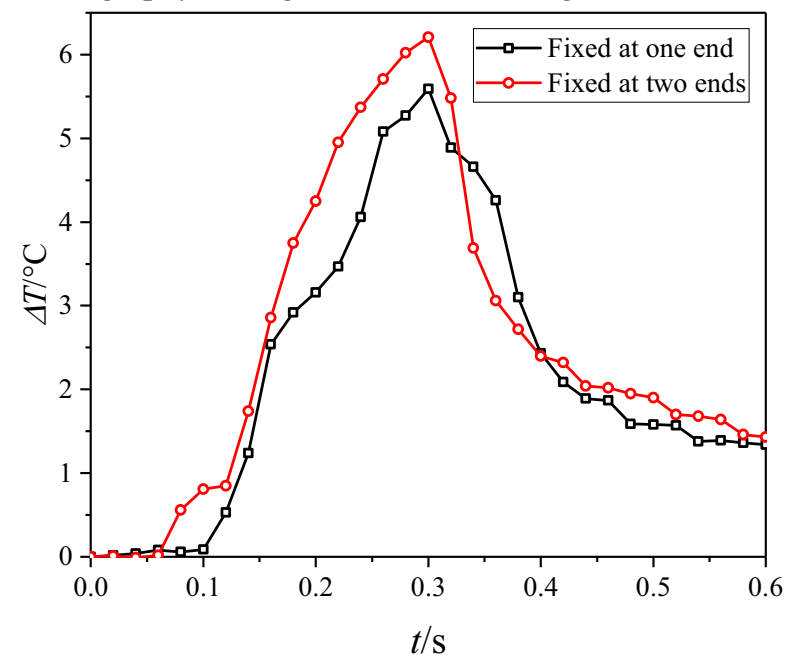

(a)

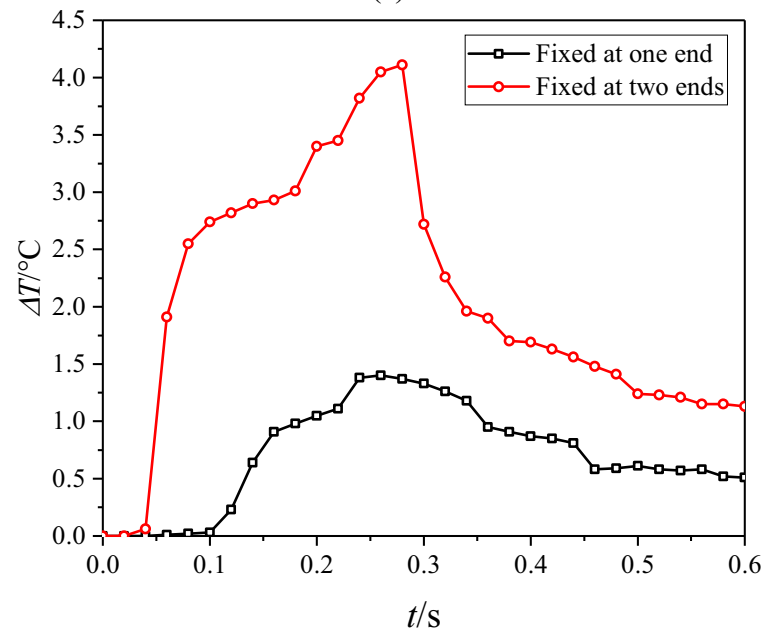

(b)

Fig. 8. The temperature rise curves of the crack area under different fixing methods: (a) $1 \#$ and (b) $2 \#$

\section{Conclusion}

Through different fixing methods to simulate the fixing methods of different structures, the ultrasonic infrared thermography testing of the samples with different length cracks are carried out. It can be found that the more the boundary of the structure is fixed during the installation process, the more the degree of freedom is restricted, and the better the detection results. The work is conducive to the optimization and selection of parameters for ultrasonic infrared thermography testing of structures under different fixed methods.

\section{References}

1. http://finance.sina.com.cn/wm/2020-06-02/dociircuyvi6297139.shtml

2. https://mil.eastday.com/a/170921094418695.html.

3. https://baijiahao.baidu.com/s?id=161929455154195 5092
4. X. Wu, Y. Yu, L. Lv. Review on Non-Destructive Detection of Inner Defects of Object. Laser \& Optoelectronics Progress, 50, 040002(2013)

5. R. K. Mishra . Nandi Vaishakhi . R. R. Bhatt, Thermo-mechanical Fatigue Failure of a LowPressure Turbine Blade in a Turbofan Engine. J Fail. Anal. and Preven, 18, 233-240(2018)

6. S. Liu, B. Hu, Z. He, Y. Li. Comparison and Evaluation on the Non-destructive Testing Methods for the Wind Turbine Blades Defects. Nondestructive Testing, 37(3): 47-50 (2015)

7. K. Song, C. Wang, L. Wang, C. Wang, Y. Li, Z. Zhao. Design and Experimental Study of Automated Eddy Current Testing System for Turbine Blade Crack. Non-destructive Testing, 61(19): 45-49 (2018)

8. A. Dyrwal, M. Meo, F. Ciampa. Nonlinear aircoupled thermosonics for fatigue micro-damage detection and localisation. NDT\&E International, 97, 59-67 (2018)

9. Y. Jia, W. Zhang, R. Zhang, J. Liu, Z. Yang. Simulation of Surface Crack Detection of TC4 Curvature. SURFACE TECHNOLOGY, 47(10), 302-308 (2018)

10. H. Jiang, L. Chen, Y. Wei, Q. Su, J. Xing, Application of Ultrasonic Thermography to Crack Detection of Aero-engine Blades. FAR EAST NDT, 618-621, 2018

11. F. Feng, Q. Min, C. Zhang, P. Jiang. Micro-cracks Heating Characteristics Under Ultrasonic Excitation Based on Thermal-solid Coupling Analysis. Journal of Academy of Armored Force Engineering, 27(5), 81-85 (2018)

12. F. Feng, C. Zhang, Q. Min, P. Wang. Heating characteristics of metal plate crack in sonic IR imaging. Infrared and Laser Engineering, 44(5), 1456-1461 (2015)

13. H. Liu, J. Liu, Y. Wang. Detection of contacting interface-type defects using ultrasound lock-in thermography. Optics and Precisio n Engineering, 18(3), 653-661 (2010) 